\title{
DAPT suppresses proliferation and migration of hepatocellular carcinoma by regulating the extracellular matrix and inhibiting the Hes1/PTEN/AKT/mTOR signaling pathway
}

\author{
Kaijie Qiu ${ }^{1 \#}$, Chenyang Ma ${ }^{1 \#}$, Lingchao $\mathrm{Lu}^{2}$, Jie Wang ${ }^{1}$, Baiwen Chen ${ }^{1}$, Haixiang Mao ${ }^{1}$, Yanmin Wang ${ }^{3}$, \\ Haibiao Wang ${ }^{1}$ \\ ${ }^{1}$ Department of Hepatobiliary and Pancreatic Surgery, Ningbo Medical Center Lihuili Hospital, Ningbo, China; ${ }^{2}$ Department of Common Surgery, \\ Yuyao Fourth People's Hospital, Ningbo, China; ${ }^{3}$ Department of operation room, Ningbo Medical Center Lihuili Hospital, Ningbo, China \\ Contributions: (I) Conception and design: H Wang, K Qiu; (II) Administrative support: H Wang, C Ma; (III) Provision of study materials or patients: \\ K Qiu, L Lu, Y Wang; (IV) Collection and assembly of data: C Ma, H Mao, K Qiu; (V) Data analysis and interpretation: C Ma, J Wang, B Chen; (VI) \\ Manuscript writing: All authors; (VII) Final approval of manuscript: All authors. \\ \#These authors contributed equally to this work. \\ Correspondence to: Haibiao Wang. Department of Hepatobiliary and Pancreatic Surgery, Ningbo Medical Center Lihuili Hospital, \#57 Xingning \\ Road, Yinzhou District, Ningbo 315000, China. Email: 13567878129@163.com.
}

Background: The aim of the present study was to investigate the antitumor properties of N-(N-[3,5difluorophenacetyl]-1-alanyl)-S-phenylglycine t-butyl ester (DAPT) against hepatocellular carcinoma (HCC), as well as the underlying mechanism.

Methods: Immunohistochemistry and quantitative reverse transcription polymerase chain reaction (qRTPCR) assay were used to determine the expression of Notch1 in HCC tissues. The expression of Notch1 in 3 HCC cell lines was evaluated by qRT-PCR and Western blot. The proliferation ability of cells was detected by 3-(4,5-dimethylthiazol-2-yl)-2,5-diphenyltetrazolium bromide and colony formation assays. Flow cytometry and Transwell assay were used to check the apoptosis and migration of HepG2 cells, respectively. Western blot was used to determine the expression level of Notch1, Hes1, Phosphatase and tensin homolog (PTEN), protein kinase B1 (AKT1), phosphorylated AKT1, mammalian target of rapamycin (mTOR), phosphorylated mTOR, intracellular adhesion molecule-1, vascular cell adhesion protein 1, matrix metalloproteinase (MMP)-2, MMP-9, and focal adhesion kinase in cells and tumor tissues. A HepG2 xenograft experiment was conducted to evaluate the in vivo antitumor properties of DAPT.

Results: Notch1 was found to be significantly upregulated in both HCC tissues and cell lines. DAPT significantly inhibited the proliferation and migration of HepG2 cells in a dose-dependent manner, accompanied by the suppression of Notch1/Hes1 signaling, inactivation of AKT/mTOR signaling, downregulation of MMPs, and decreased expression of adhesion molecules. The activation of Notch1/ Hes1 or AKT/mTOR signaling removed the inhibitory effect of DAPT on the proliferation and migration of HepG2 cells, as well as the inhibitory properties of DAPT on the expression of MMPs and adhesion molecules. The antitumor properties and regulatory effect of DAPT against the extracellular matrix (ECM) and Hes1/PTEN/AKT/mTOR signaling were verified by the HepG2 xenograft experiments.

Conclusions: DAPT could suppress the proliferation and migration of HCC by regulating the ECM and inhibiting the Hes1/PTEN/AKT/mTOR signaling pathway.

Keywords: Notch1; hepatocellular carcinoma; protein kinase B; mammalian target of rapamycin; N-(N-[3,5difluorophenacetyl]-1-alanyl)-S-phenylglycine t-butyl ester

Submitted Mar 12, 2021. Accepted for publication May 28, 2021.

doi: 10.21037/jgo-21-235

View this article at: http://dx.doi.org/10.21037/jgo-21-235 


\section{Introduction}

Hepatocellular carcinoma (HCC) is one of the most common malignant tumors (1). The overall survival rate of HCC patients is relatively low. In addition, in China, the survival rate during the early stage of HCC has been reported to be around $60 \%$, while the 5 -year survival rate is approximately $33 \%$ (2). Global oncology epidemiological investigations indicate that approximately 0.7 million patients are diagnosed with HCC annually, and 0.65 million patients die from HCC every year, with half from China (3). Health statistics from China's Ministry of Health indicate that HCC has a severe impact on public health owing to its secondary death rate in all types of malignant tumors (4). Therefore, it is important to investigate the pathological mechanism of HCC and develop effective therapies for its treatment.

Several molecular signaling pathways have been reported to be involved in the development and progression of HCC, including the mitogen-activated protein kinase (MAPK), Phosphatase and tensin homolog (PTEN)/mammalian target of rapamycin (mTOR)/protein kinase B (AKT), and Notch signaling pathways (5). The Notch signaling pathway is an important signaling cassette that exists in multiple species, and consists of the membrane ligand, receptor, and downstream molecules (6). The abnormal activation of the Notch signaling pathway has been reported to be closely related to the pathogenesis of HCC, in which Notch1 is a vital regulator that is claimed to be involved in epithelial-mesenchymal transition, embryo development, cellular proliferation and differentiation, and the repair of impaired organs, as well as the pathogenesis of malignant tumors (7). In HCC tissues, Notch 1 is found to be significantly upregulated (8). Further functional investigations indicate that the expression of cell circlerelated proteins, such as cyclin $\mathrm{A}, \mathrm{E} 2$, and $\mathrm{D} 1$, can be significantly suppressed by Notch1 (9). Increasing evidence has suggested that Notch1 facilitates the development of HCC. Zhang et al. reported that Notch1, 3, and 4 were found to be highly expressed in HCC tissues, which played an important role in the prognosis of HCC (10). Villanueva et al. reported that the Notch signaling is activated in human HCC and contributes to tumor formation in animal models (11). In addition, the suppression of the Notch1 signaling pathway has been reported to be involved in the mechanism of multiple anti-HCC molecules, such as ICBP90, LINC00261, and salidroside (12-14). Therefore, blocking the Notch1 signaling pathway might be an effective method for the treatment of HCC. N-(N-[3,5difluorophenacetyl]-1-alanyl)-S-phenylglycine t-butyl ester (DAPT) is an inhibitor of Notch1 that is widely used for the verification of the involvement of the Notch1 signaling pathway as a blocker $(15,16)$. DAPT is an inhibitor of $\gamma$-Secretase and inhibits the segmentation processing of S3 domain in Notch, which release the Notch intracellular domain to further activate the transcription of downstream genes. DAPT has been widely used for the inhibition of Notch pathway (doi: 10.1002/jnr.21368). Although the antitumor property of DAPT in hepatocellular carcinoma has already been reported (30309512), the underlying mechanism have not been illustrated clearly. Therefore, in the present study, we explored the potential therapeutic properties of DAPT against HCC by investigating regulation of DAPT on Hes1/PTEN/AKT/mTOR signaling pathway. We present the following article in accordance with the ARRIVE reporting checklist (available at http://dx.doi.org/10.21037/jgo-21-235).

\section{Methods}

\section{Tissues, cells, animals, and treatments}

Ten pairs of HCC tissues and para-carcinoma tissues were obtained from Ningbo Medical Center Lihuili Hospital (Ningbo, China), All procedures performed in this study involving human participants were in accordance with the Declaration of Helsinki (as revised in 2013). The study was approved by Ethics Committee Board of Ningbo Medical Center Lihuili Hospital (NO: KY2020PJ190). Because of the retrospective nature of the study, the requirement for informed consent was waived. HCC cell lines, including HepG2, MHCC97H, and HCCLM3, as well as the normal hepatocyte L-02, were purchased from American Type Culture Collection (Manassas, VA, USA), which were cultured in Dulbecco's modified Eagle's medium (Gibco, Grand Island, NY, USA) containing 10\% fetal bovine serum and $0.1 \%$ penicillin and streptomycin at $37{ }^{\circ} \mathrm{C}$. Fifteen BABL/c nude mice (T cell defect, 6-8 weeks old, 18-23 g) were obtained from Beijing Vital River Laboratory Animal Technology (Beijing, China) for the in vivo xenograft experiment, the experimental unit of which was a single animal. The animals had access to food and water during the experiments, Experiments were performed under a project license (NO: 2019-381) granted by the Animal Ethics committee of Ningbo University School of Medicine, in compliance with institutional 
Table 1 Sequences of primers for Notch1 and GAPDH

\begin{tabular}{lcc}
\hline Primer name & Primer sequence (5'-3') & Primer length (bp) \\
\hline Notch1 forward & GAGGCGTGGCAGACTATG & 18 \\
Notch1 reverse & CTTGTACTCCGTCAGCGTG & 19 \\
GAPDH forward & CAATGACCCCTTCATTGACC & 20 \\
GAPDH reverse & GAGAAGCTTCCCGTTCTCAG & 20 \\
\hline
\end{tabular}

guidelines for the care and use of animals. The chemical molecules (DAPT and MHY-1485) were purchased from MedChemExpress (Monmouth Junction, NJ, USA).

\section{Immunobistochemical (ICH) assay}

ICH assay was performed on tissues. The tissues were fixed with $10 \%$ neutral buffered formalin solution (Bio-Optica, Milan, Italy), and were then embedded with paraffin to obtain 4- $\mu$ m-thick sections, which were airdried overnight and fixed in acetone for $10 \mathrm{~min}$. After airdrying for another $20 \mathrm{~min}$, the sections were incubated with $0.2 \%$ galantine (Sigma, San Francisco, CA, USA) and 0.2\% Triton X-100 in phosphate-buffered saline (PBS), and further blocked with an antibody diluent (Dako, Copenhagen, Denmark) for $1 \mathrm{~h}$ at room temperature. Subsequently, the slides were deparaffinized and stained with primary antibody against Notch1 (1:100; Cell Signaling Technology, Boston, MA, USA) at $4^{\circ} \mathrm{C}$ overnight. After washing using PBS buffer, secondary antibodies (1:100; Cell Signaling Technology, USA) were added, and the sections were incubated for $1 \mathrm{~h}$ with Hoechst 33342 (Sigma, USA) at room temperature. After incubation, the sections were analyzed with LSM710 confocal microscopes (Zeiss, Oberkochen, Germany).

\section{Quantitative reverse transcription polymerase chain reaction (qRT-PCR)}

TRIzol reagent (Invitrogen, Carlsbad, CA, USA) was used to extract total RNA from the cells and tissues, and the RNAs were further transcribing into cDNA using the RevertAid First Strand cDNA Synthesis Kit (Thermo Scientific, Waltham, MA, USA), according to manufacturer's instructions. Subsequently, cDNA was applied for the quantitative real-time PCR in a $20-\mu \mathrm{L}$ PCR reaction system containing $10 \mu \mathrm{L} 2 \times$ Power SYBR Green Master Mix (Invitrogen, USA). GAPDH was used to normalize the relative expression of target genes, which was calculated using the $2^{-\Delta \Delta \mathrm{Ct}}$ method. Primer sequences are shown in Table 1.

\section{Western blot assay}

Cells or tumor tissues were lysed using cellular lysis buffer (Thermo Scientific, USA) to extract total proteins, which were loaded and separated using sodium dodecylsulfatepolyacrylamide gel electrophoresis. The separated proteins were then transferred onto a polyvinylidene difluoride membrane, followed by incubation with $5 \%$ bicinchoninic acid solution to remove non-specific binding proteins. Subsequently, the membrane was incubated with a primary antibody against Notch1 (1:1,000; Cell Signaling Technology, USA), Hes1 (1:1,000; Cell Signaling Technology, USA), PTEN (1:1,000; Cell Signaling Technology, USA), phosphorylated AKT1 (p-AKT1; 1:1,000; Cell Signaling Technology, USA), AKT1 (1:1,000; Cell Signaling Technology, USA), AKT1 (1:1,000; Cell Signaling Technology, USA), phosphorylated mTOR (p-mTOR; 1:1,000; Cell Signaling Technology, USA), mTOR (1:1,000; Cell Signaling Technology, USA), intracellular adhesion molecule-1 (ICAM-1; 1:1,000; Cell Signaling Technology, USA), vascular cell adhesion protein 1 (VCAM-1; 1:1,000; Cell Signaling Technology, USA), matrix metalloproteinase (MMP)-2 (1:1,000; Cell Signaling Technology, USA), MMP-9 (1:1,000; Cell Signaling Technology, USA), focal adhesion kinase (FAK; 1:1,000; Cell Signaling Technology, USA) or GAPDH $(1: 1,000$; Cell Signaling Technology, USA), followed by incubation with a horseradish peroxidase-conjugated secondary antibody (1:1,000; Cell Signaling Technology, USA) at room temperature for 1-2 $\mathrm{h}$, all antibodies used are shown in Table 2. Finally, the blots were incubated with enhanced chemiluminescence reagents (Thermo Scientific, USA) and visualized using an Image J software (National Institutes of 
Table 2 Antibodies used in the Western blot assay.

\begin{tabular}{llc}
\hline Antibodies & \multicolumn{1}{c}{ Manufacturer } & Dilution ratio \\
\hline Anti-Notch1 & Cell Signaling Technology & $1: 1,000$ \\
Anti-Hes1 & Cell Signaling Technology & $1: 1,000$ \\
Anti-PTEN & Cell Signaling Technology & $1: 1,000$ \\
Anti-p-AKT1 & Cell Signaling Technology & $1: 1,000$ \\
Anti-AKT1 & Cell Signaling Technology & $1: 1,000$ \\
Anti-p-mTOR & Cell Signaling Technology & $1: 1,000$ \\
Anti-mTOR & Cell Signaling Technology & $1: 1,000$ \\
Anti-ICAM-1 & Cell Signaling Technology & $1: 1,000$ \\
Anti-VCAM-1 & Cell Signaling Technology & $1: 1,000$ \\
Anti-MMP-2 & Cell Signaling Technology & $1: 1,000$ \\
Anti-MMP-9 & Cell Signaling Technology & $1: 1,000$ \\
Anti-FAK & Cell Signaling Technology & $1: 1,000$ \\
Anti-GAPDH & Cell Signaling Technology & $1: 1,000$ \\
\hline
\end{tabular}

Health, Bethesda, Maryland, USA).

\section{3-(4,5-Dimethylthiazol-2-yl)-2,5-diphenyltetrazolium bromide (MTT) assay}

MTT assay was used to evaluate the proliferation ability of HCC cells. In brief, $10 \mu \mathrm{L}$ of $5 \mathrm{mg} / \mathrm{mL}$ MTT solution was added to the medium (Thermo Scientific, USA), followed by incubation for $4 \mathrm{~h}$. Subsequently, approximately $200 \mu \mathrm{L}$ of formazan in dimethylsulfoxide (Genview, Beijing, China) was added to the medium. Lastly, absorbance (optical density value) at $490 \mathrm{~nm}$ was read using a microplate reader (BMG LABTECH, Offenburg, Germany).

\section{Colony formation assay}

Cells at the exponential phase were seeded onto 6-well plates at a density of 500 cells/well with 3 replicates. After incubating for 10-14 days, the cells were fixed with $4 \%$ paraformaldehyde (Solarbio Science \& Technology, Beijing, China) for $15 \mathrm{~min}$ and stained with Giemsa (Solarbio Science \& Technology, Beijing, China) for $30 \mathrm{~min}$ at room temperature. After washing the cells several times, the number of cell colonies was calculated.

\section{Flow cytometry for the apoptosis analysis}

The treated cells were digested and resuspended using $400 \mu \mathrm{L}$ of $1 \times$ binding buffer, and approximately $100 \mu \mathrm{L}$, cell suspension was added to a $5-\mathrm{mL}$ flow tube, followed by $5 \mu \mathrm{L}$ Annexin V/Alexa Fluor647 (Beijing 4A Biotech, Beijing, China). After incubation at room temperature for $5 \mathrm{~min}$ in the dark, the samples were incubated with $10 \mu \mathrm{L}$ propidium iodide and $400 \mu \mathrm{L}$ PBS. Finally, the Accuri C6 (BD Biosciences, Franklin Lakes, NJ, USA) flow cytometer was used to detect and analyze the fluorescence intensity of the cells.

\section{Transwell migration assay}

The upper chamber of the well plate was planted with $1 \times 10^{5}$ cells per well and filled with $100 \mu \mathrm{L}$ of serum-free medium. Subsequently, approximately $600 \mu \mathrm{L}$ of complete medium was added to the lower chamber, followed by incubation for $24 \mathrm{~h}$ at $37^{\circ} \mathrm{C}$. The residual cells in the upper chamber were removed and the cells in the lower chamber were the migrated cells, which were fixed with $4 \%$ paraformaldehyde and stained with $0.1 \%$ crystal violet solution. Finally, the migrated cells were photographed using an inverted 
fluorescence microscope (Magnification: 200×, Olympus, Tokyo, Japan).

\section{Transfection}

The pcDNA3.1-Notch1 and pcDNA3.1-Hes1 were designed and established by Genscript (Nanjing, China), and were transfected into HepG2 cells, along with the transfection reagent (Lipofectamine 3000; Thermo Fisher, Waltham, Massachusetts, USA) to obtain the Notch1- and Hes1-overexpressed HepG2 cells.

\section{Xenograft experiments}

For the in vivo xenograft experiment, we used female nude mice, the cells were randomly divided into 5 groups, using a computer-generated random numbers table as follows: control, DAPT+pcDNA3.1-negative control (NC), DAPT+pcDNA3.1-Notch1, DAPT+pcDNA3.1Hes1, and DAPT+MHY-1485. No confounders were involved. The nude mice in the control group were planted with naive HepG2 cells, followed by being administered subcutaneously with normal saline for 3 weeks. The animals in the DAPT+pcDNA3.1-NC group were transplanted with pcDNA3.1-NC-transfected HepG2 cells and treated subcutaneously with $10 \mathrm{mg} / \mathrm{kg} /$ day DAPT for 3 weeks (17). HepG2 cells transfected with pcDNA3.1Notch1 and DAPT+pcDNA3.1-Hes1, were planted into the nude mice, followed by being administered subcutaneously with $10 \mathrm{mg} / \mathrm{kg} /$ day DAPT for 3 weeks, respectively. In the DAPT+MHY-1485 group, HepG2 cells were pretreated with $10 \mu \mathrm{M}$ mTOR activator MHY-1485 for $6 \mathrm{~h}$ and then transplanted into the nude mice (18), which were further administered subcutaneously with $10 \mathrm{mg} / \mathrm{kg} /$ day DAPT for 3 weeks. For each group, 3 animals were utilized. All the treatments were performed until the volume of the tumor reached $100 \mathrm{~mm}^{3}$. Tumor volume was measured and calculated according to the following formula: $\mathrm{V}=\mathrm{L} \times \mathrm{W} 2 / 2$, where $\mathrm{V}$ represents volume $\left(\mathrm{mm}^{3}\right), \mathrm{L}$ represents the greatest diameter $(\mathrm{mm})$, and $\mathrm{W}$ represents the lowest diameter $(\mathrm{mm})$. The animals were killed at the end of the experiment, and the tumors were pictured and weighed. The Sample size was determined based on the number of experimental groups and different time points of analysis. For all allocation, only designer was aware of the group allocation at the different stages of the experiment.
No criteria used for including and excluding animals as there were no exclusions.

\section{Statistical analysis}

All experiments were repeated independently 3 times, and data are shown as mean \pm standard deviations. Statistical analyses were performed using SPSS version 24.0 (IBM, Armonk, NY, USA), and significant differences were analyzed by one-way analysis of variance coupled with posthoc comparisons. Student's t-test was used to analyze the difference between groups. Significance was defined as $\mathrm{P}<0.05$.

\section{Results}

\section{High expression of Notch1 in HCC tissues and cell lines}

To investigate the function of Notch1 in the development and progression of HCC, we analyzed the expression of Notch1 in both HCC tissues from patients and HCC cell lines. As shown in Figure 1A,B, ICH and qRT-PCR assay revealed that, compared with para-carcinoma tissues, Notch1 was found to be significantly upregulated in HCC tissues from patients $(\mathrm{P}<0.01)$. Subsequently, we further detected the relative expression level of Notch1 in HCC cell lines and normal hepatocytes. As shown in Figure 1C and $\mathrm{D}$, the expression level of Notch1 was significantly elevated in HCC cell lines (all $\mathrm{P}<0.05$ ). In addition, in the 3 HCC cell lines, the highest expression of Notch1 was found in HepG2 cells. Therefore, HepG2 cells were used in our subsequent experiments to investigate the function of Notch1 in HCC.

\section{Suppression of proliferation and migration ability of HepG2 cells by DAPT}

To explore the function of DAPT, an inhibitor of Notch1, on the development of HCC, HepG2 cells were treated with different dosages of DAPT $(25,50$, and $100 \mu M, n=3)$, with HepG2 cells incubated with blank medium as the control group. As shown in Figure $2 A$, the cell viability of HepG2 cells was significantly inhibited by the introduction of DAPT in a dose-dependent manner (all $\mathrm{P}<0.05$ ). Compared with the control, in the colony formation assay, the colony number was found to be significantly suppressed 
A
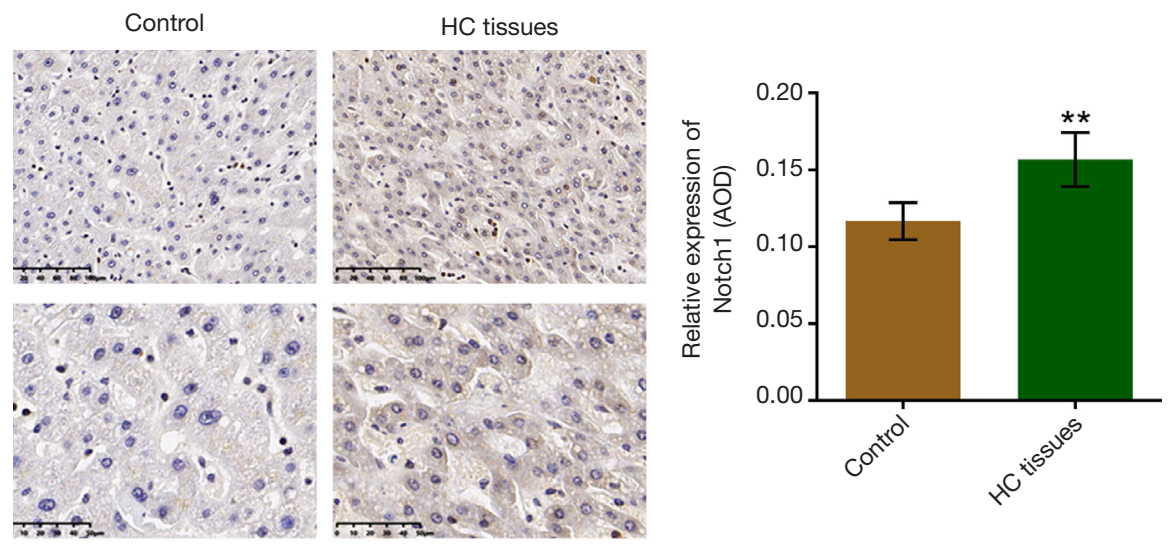

B

C
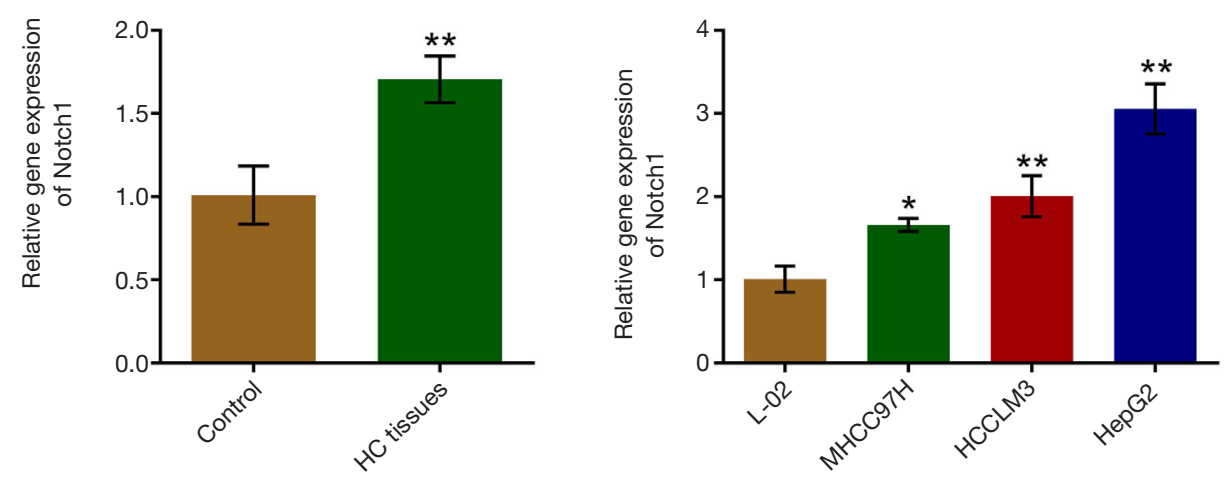

$\mathrm{D}$
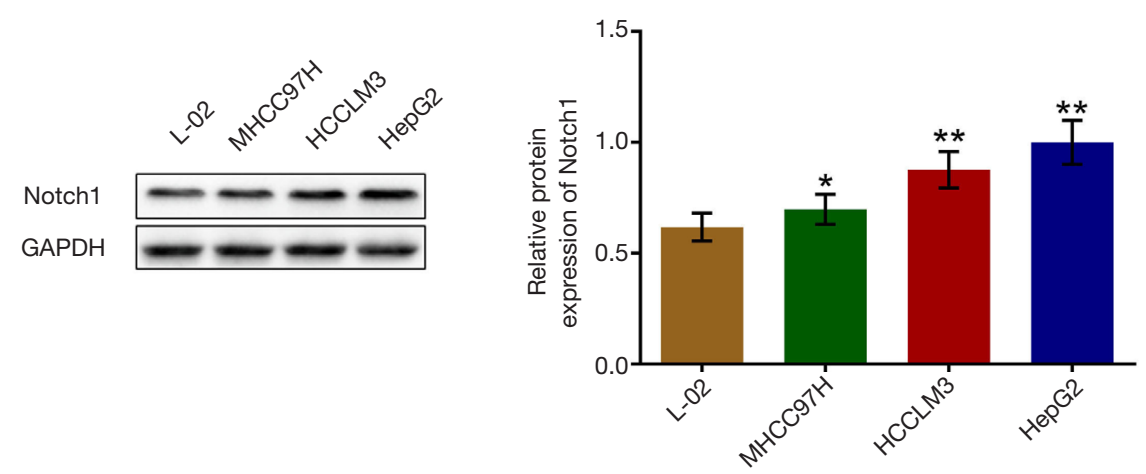

Figure 1 Notch1 was up-regulated in both hepatocellular carcinoma (HCC) tissues and cell lines. (A) Expression of Notch1 in HCC tissues was determined by immunohistochemical assay, Scale bar (200x): $20 \mu \mathrm{m}$. (B,C) Expression of Notch1 in HCC tissues and cell lines was detected by quantitative reverse transcription polymerase chain reaction assay. (D) Expression of Notch1 in HCC cell lines was evaluated by Western blot assay. ${ }^{*} \mathrm{P}<0.05$ vs. control; ${ }^{* *} \mathrm{P}<0.01$ vs. control.

in the 50 and $100 \mu \mathrm{M}$ DAPT groups $(\mathrm{P}<0.01)$ (Figure $2 B$ ). As shown in Figure $2 C$, compared with the control, the apoptotic rate of HepG2 cells increased from $5.45 \%$ to $15.71 \%$ and $18.29 \%$ in the 50 and $100 \mu \mathrm{M}$ DAPT groups, respectively $(\mathrm{P}<0.01)$. Finally, in the Transwell assay, the number of migrated cells was significantly decreased by the treatment of DAPT in a dose-dependent manner $(\mathrm{P}<0.05)$ (Figure 2D). These data indicated that the proliferation and migration ability of HepG2 cells were significantly inhibited by DAPT. 

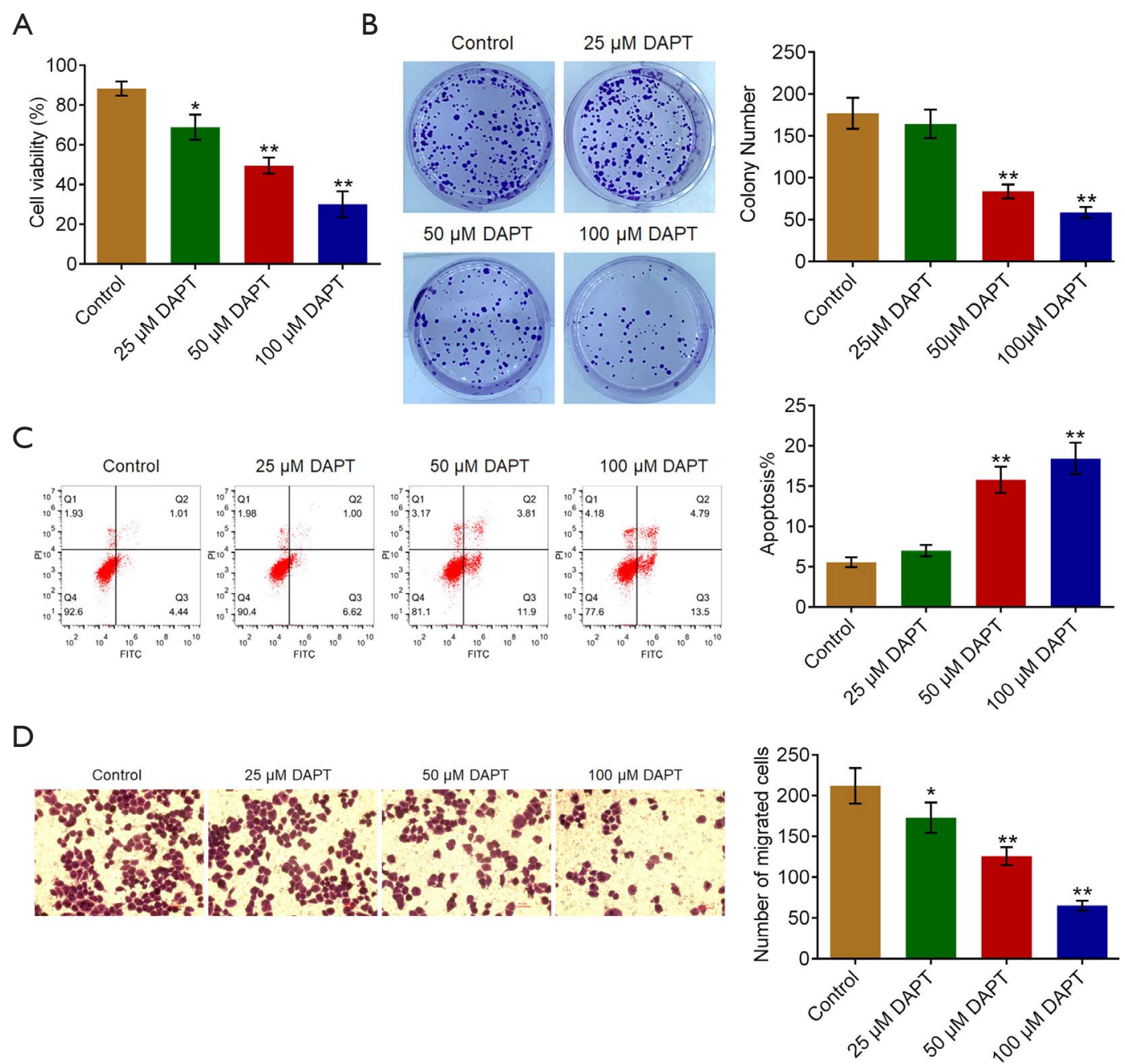

Figure 2 Proliferation and migration of HepG2 cells were suppressed, and the apoptotic rate of HepG2 cells was elevated by N-(N[3,5-difluorophenacetyl]-1-alanyl)-S-phenylglycine t-butyl ester in a dose-dependent manner. (A) Cell viability was evaluated by 3-(4,5-dimethylthiazol-2-yl)-2,5-diphenyltetrazolium bromide assay; (B) proliferation ability was determined by colony formation assay; (C) apoptosis was detected by flow cytometry; (D) migration ability of HepG2 cells was checked by Transwell assay, Scale bar: $40 \mu \mathrm{m}$. ${ }^{*} \mathrm{P}<0.05$ vs. control; ${ }^{* *} \mathrm{P}<0.01$ vs. control.

\section{Effects of DAPT on the Hes1/PTEN/AKT/mTOR signaling pathway, extracellular matrix (ECM), and adbesion molecules}

We further investigated the effects of DAPT on the downstream signaling pathway of Notch 1 and the protection of the ECM. As shown in Figure 3, the expression of Notch1 and Hes1 was significantly inhibited, and the expression of PTEN was significantly elevated by the introduction of DAPT in a dose-dependent manner $($ all $\mathrm{P}<0.05)$, indicating negative regulation of
DAPT on the Notch1/Hes1/PTEN signaling pathway. Subsequently, we found that $\mathrm{p}-\mathrm{AKT} 1$ and $\mathrm{p}-\mathrm{mTOR}$ were significantly downregulated by the treatment of DAPT in a dose-dependent manner (all $\mathrm{P}<0.05$ ), indicating an inhibitory effect of DAPT on the AKT/mTOR signaling pathway. In addition, the expression of FAK, ICAM-1, and VCAM-1, was found to be significantly suppressed by DAPT $(\mathrm{P}<0.01)$. Finally, MMP-2 and MMP-9 were significantly downregulated by the treatment of DAPT in a dose-dependent manner $(\mathrm{P}<0.01)$, indicating protective 

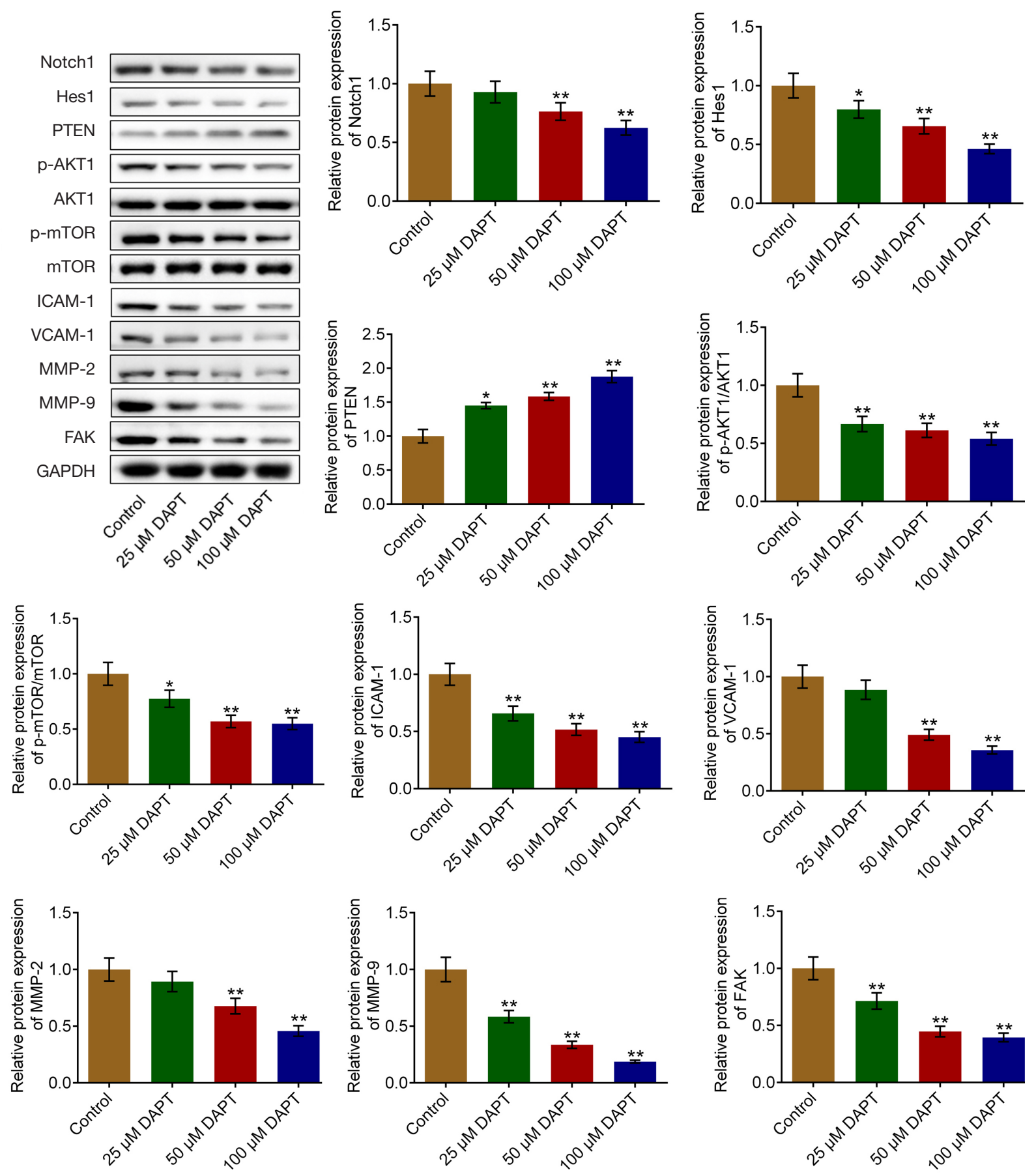

Figure $3 \mathrm{~N}-(\mathrm{N}-[3,5-D i f l u o r o p h e n a c e t y l]-1-a l a n y l)-S-p h e n y l g l y c i n e ~ t-b u t y l$ ester (DAPT) significantly inhibited Notch1/Hes1/ Phosphatase and tensin homolog (PTEN) and protein kinase B (AKT)/mammalian target of rapamycin (mTOR) signaling pathways, and the expression of matrix metalloproteinases (MMPs) and adhesion molecules. Expression level of Notch1, Hes1, PTEN, AKT1, phosphorylated AKT1, mTOR, phosphorylated mTOR, intracellular adhesion molecule 1, vascular cell adhesion protein 1, MMP-2, MMP-9, and focal adhesion kinase in the DAPT-treated HepG2 cells was evaluated by Western blot assay. ${ }^{*} \mathrm{P}<0.05$ vs. control; ${ }^{* *} \mathrm{P}<0.01$ vs. control. 
properties of DAPT on the ECM.

\section{Abolition of DAPT biofunction by activation of the Hes1/ PTEN/AKT/mTOR signaling pathway}

To verify the involvement of the Hes1/PTEN/AKT/ mTOR signaling pathway in the antitumor function of DAPT, Notch1-overexpressed, Hes1-overexpressed HepG2 cells, and MHY-1485, an mTOR activator, were introduced. As shown in Figure 4A,B, cell viability and the colony number were significantly suppressed in the DAPT-treated HepG2 cells transfected with pcDNA3.1$\mathrm{NC}$; these were significantly reversed in the DAPT-treated Notch1-overexpressed and Hes1-overexpressed HepG2 cells, or DAPT and MHY-1485-co-treated HepG2 cells (all $\mathrm{P}<0.01$ ). As shown in Figure $4 C$, the apoptotic rate in the control, DAPT+pcDNA3.1-NC, DAPT+pcDNA3.1Notch1, DAPT+pcDNA3.1-Hes1, and DAPT+MHY-1485 groups was $7.29 \%, 25.85 \%, 14.86 \%, 15.61 \%$, and $14.49 \%$, respectively (all $\mathrm{P}<0.01$ ). Finally, in the Transwell assay, the number of migrated cells was significantly inhibited by the treatment of DAPT (Figure 4D), which was significantly elevated in the DAPT-treated HepG2 cells transfected with pcDNA3.1-NC. This was significantly reversed in the DAPT-treated Notch1-overexpressed and Hes1overexpressed HepG2 cells or DAPT and MHY-1485-cotreated HepG2 cells (all $\mathrm{P}<0.01$ ).

\section{Abolishment of the effects of DAPT on the Hes1/PTEN/ AKT/mTOR signaling pathway, ECM, and adbesion molecules by activation of the Hes1/PTEN/AKT/mTOR signaling pathway}

As shown in Figure 5, compared with the control, the expression of Notch1 and Hes1 was significantly inhibited and the expression of PTEN was significantly elevated in DAPT-treated HepG2 cells transfected with pcDNA3.1-NC; this was reversed in DAPT-treated Notch1-overexpressed and Hes1-overexpressed HepG2 cells or DAPT and MHY-1485-co-treated HepG2 cells $(\mathrm{P}<0.01$ vs. control, $\mathrm{P}<0.05$ vs. $\mathrm{DAPT}+\mathrm{pcDNA} 3.1-\mathrm{NC}$, $)$. Subsequently, the downregulated p-AKT1 and p-mTOR induced by DAPT was significantly upregulated in DAPTtreated Notch1-overexpressed and Hes1-overexpressed HepG2 cells or DAPT and MHY-1485-co-treated HepG2 cells (all $\mathrm{P}<0.01)$. In addition, the suppressed expression of FAK, ICAM-1, and VCAM-1 in HepG2 cells induced by DAPT was significantly increased in DAPT-treated
Notch1-overexpressed and Hes1-overexpressed HepG2 cells or DAPT and MHY-1485-co-treated HepG2 cells (all $\mathrm{P}<0.01$ ). Finally, the expression of MMP-2 and MMP9 was significantly inhibited by the treatment of DAPT, which was significantly reversed in DAPT-treated Notch1overexpressed and Hes1-overexpressed HepG2 cells or DAPT and MHY-1485-co-treated HepG2 cells (all $\mathrm{P}<0.01$ ).

Antitumor effects of DAPT by regulating the Hes1/PTEN/ AKT/mTOR signaling pathway and protecting the ECM in vivo

To verify the antitumor properties, as well as the underlying mechanism, of DAPT on HCC, xenograft experiments were performed $(\mathrm{n}=3)$. As shown in Figure 6, tumor volume and weight were significantly decreased in the DAPT+pcDNA3.1-NC group, and significantly elevated in the DAPT+pcDNA3.1-Notch1, DAPT+pcDNA3.1Hes1, and DAPT + MHY-1485 groups (all $\mathrm{P}<0.01$ ). These data indicated that DAPT might exert antitumor properties by inhibiting the Hes1/PTEN/AKT/mTOR signaling pathway. We further verified the expression of related proteins in the tumors. As shown in Figure 7, compared with control, the expression of Notch 1 and Hes1 was suppressed; the expression of PTEN increased; the AKT/ mTOR signaling pathway was activated, and the expression of FAK, ICAM-1, VCAM-1, MMP-2, and MMP-9 was downregulated in the DAPT+pcDNA3.1-NC group; these were all reversed in the DAPT+pcDNA3.1-Notch1, DAPT+pcDNA3.1-Hes1, and DAPT+MHY-1485 groups (all $\mathrm{P}<0.01$ ). These data confirmed that the antitumor effect of DAPT was related to the regulation of the Hes1/PTEN/ AKT/mTOR signaling pathway, ECM, and the expression of adhesion molecules.

\section{Discussion}

The Notch signaling pathway has been reported to be involved in the pathogenesis of multiple types of malignant tumors, including HCC (19). The basic helixloop-helix protein family is one of the main downstream transcriptional factors of notch signaling, including the Hes family, Homocysteine-Induced Endoplasmic Reticulum Protein (HERP) family, and YRPW motif (HEY) family (20-22). Hes inhibits the transcription of target genes by binding with the promoter of specific differentiation effector gene, and plays an important role in Notch signal transduction. The Notch/Hes signaling pathway has 
A

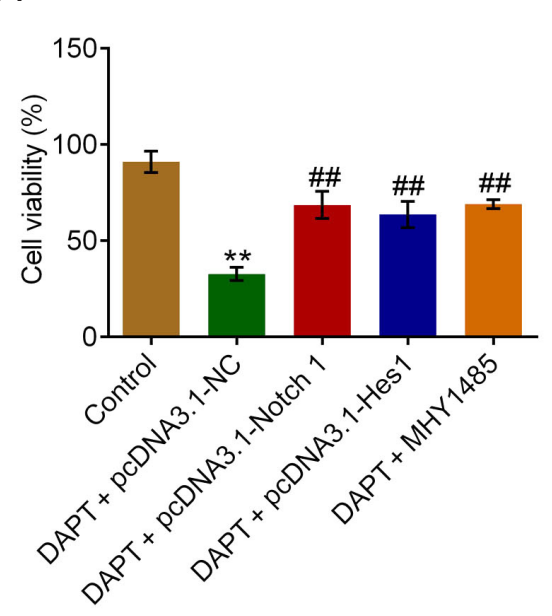

B

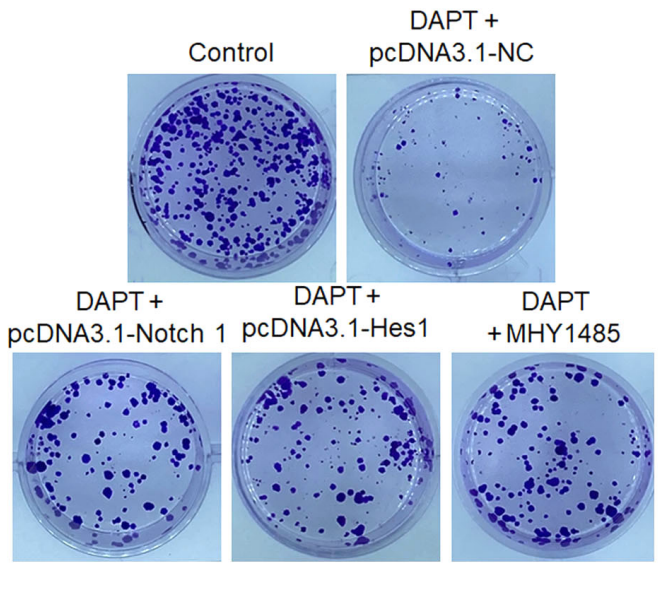

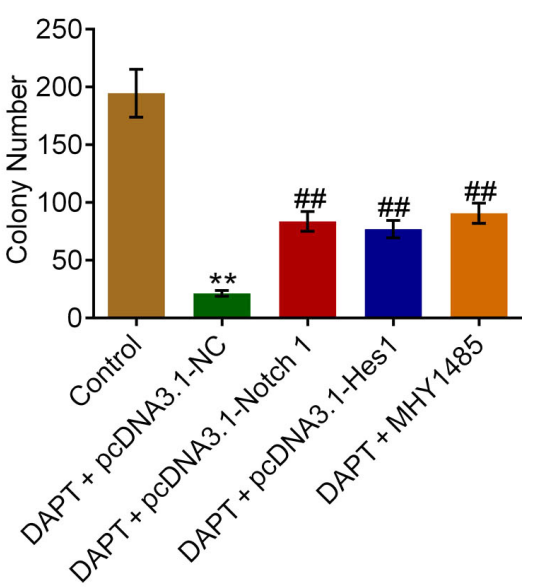

$\mathrm{DAPT}+$

C

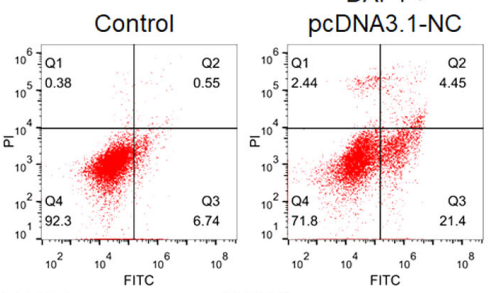

DAPT +

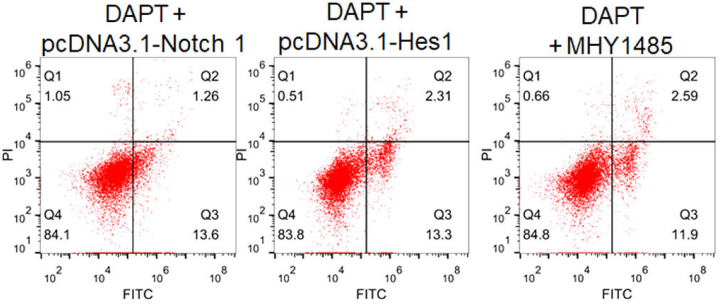

$\mathrm{DAPT}+$

D
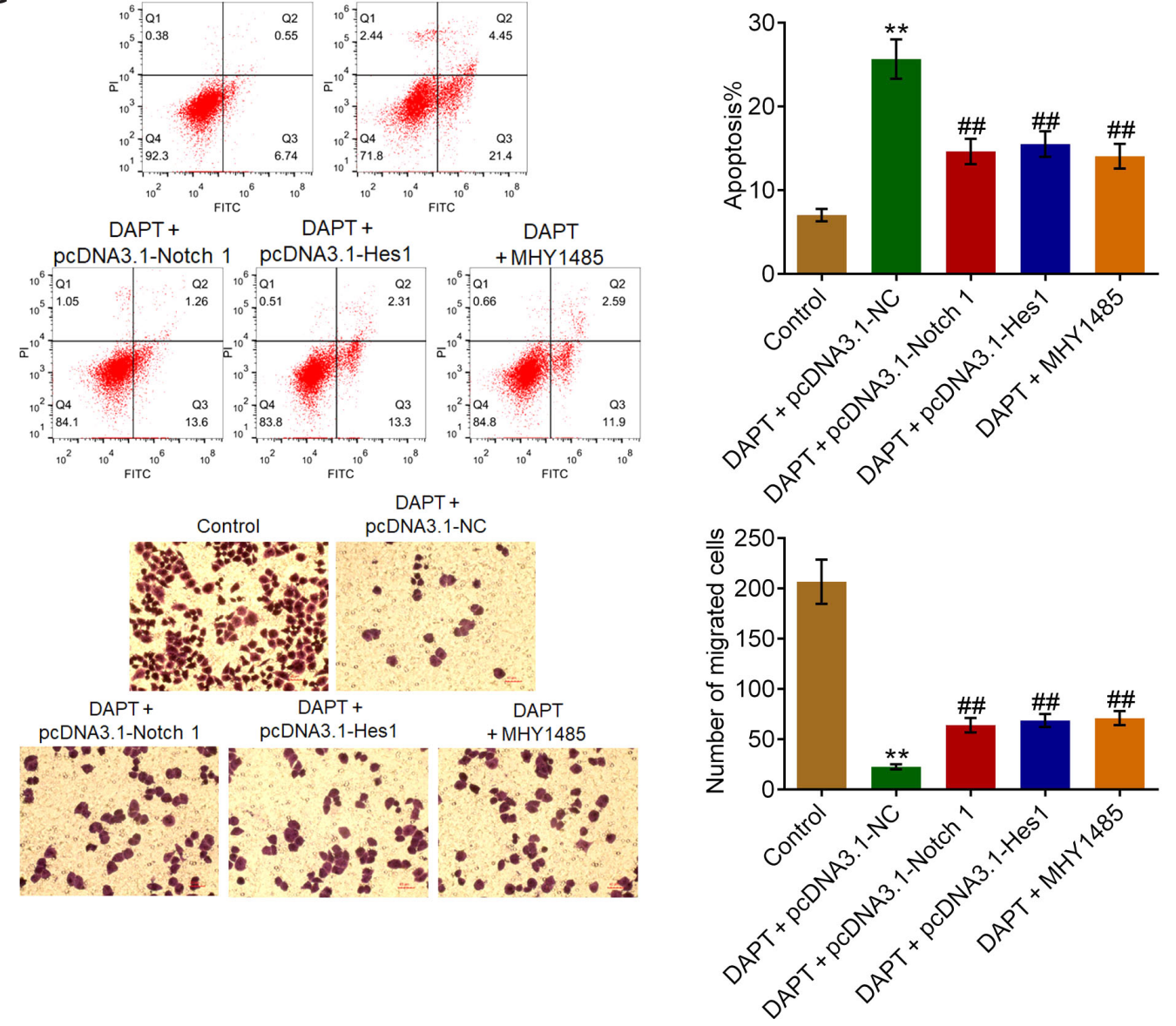

Figure 4 Regulatory effect of N-(N-[3,5-difluorophenacetyl]-1-alanyl)-S-phenylglycine t-butyl ester (DAPT) on the proliferation, apoptosis, and migration of HepG2 cells was abolished by the activation of Notch1/Hes1 and protein kinase B(AKT)/mammalian target of rapamycin (mTOR) signaling. (A) Cell viability was evaluated by 3-(4,5-dimethylthiazol-2-yl)-2,5-diphenyltetrazolium bromide assay. (B) Proliferation ability was determined by colony formation assay. (C) Apoptosis was detected by flow cytometry. (D) Migration ability of HepG2 cells was determined by Transwell assay, Scale bar: $40 \mu \mathrm{m}$. ${ }^{* *} \mathrm{P}<0.01$ vs. control, ${ }^{* \#} \mathrm{P}<0.01 v s$. DAPT+pcDNA3.1- negative control (NC). 

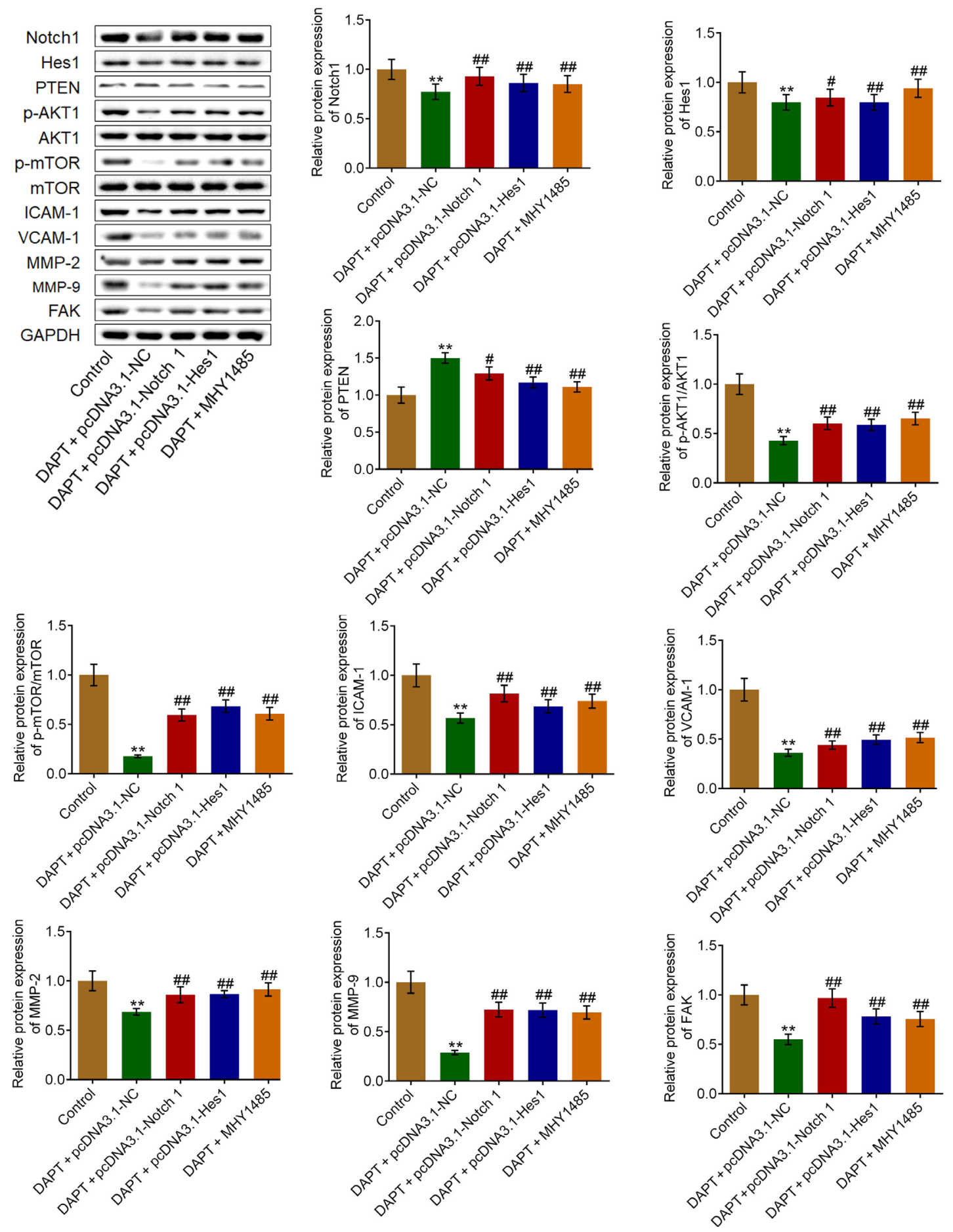

Figure 5 Effects of N-(N-[3,5-difluorophenacetyl]-1-alanyl)-S-phenylglycine t-butyl ester (DAPT) on the Hes1/Phosphatase and tensin homolog (PTEN)/protein kinase B(AKT) /mammalian target of rapamycin (mTOR) signaling pathway, ECM, and adhesion molecules were abolished by activating the Hes1/PTEN/AKT/mTOR signaling pathway. Expression level of Notch1, Hes1, PTEN, AKT1, phosphorylated AKT1, mTOR, phosphorylated mTOR, intracellular adhesion molecule 1, vascular cell adhesion protein 1, matrix metalloproteinase (MMP)-2, MMP-9, and focal adhesion kinase in the DAPT-treated HepG2 cells was evaluated by Western blot assay. ${ }^{* *} \mathrm{P}<0.01$ vs. control; ${ }^{\#} \mathrm{P}<0.05$ vs. DAPT+pcDNA3.1-negative control $(\mathrm{NC}) ;{ }^{\# \#} \mathrm{P}<0.01$ vs. DAPT+pcDNA3.1-NC. 

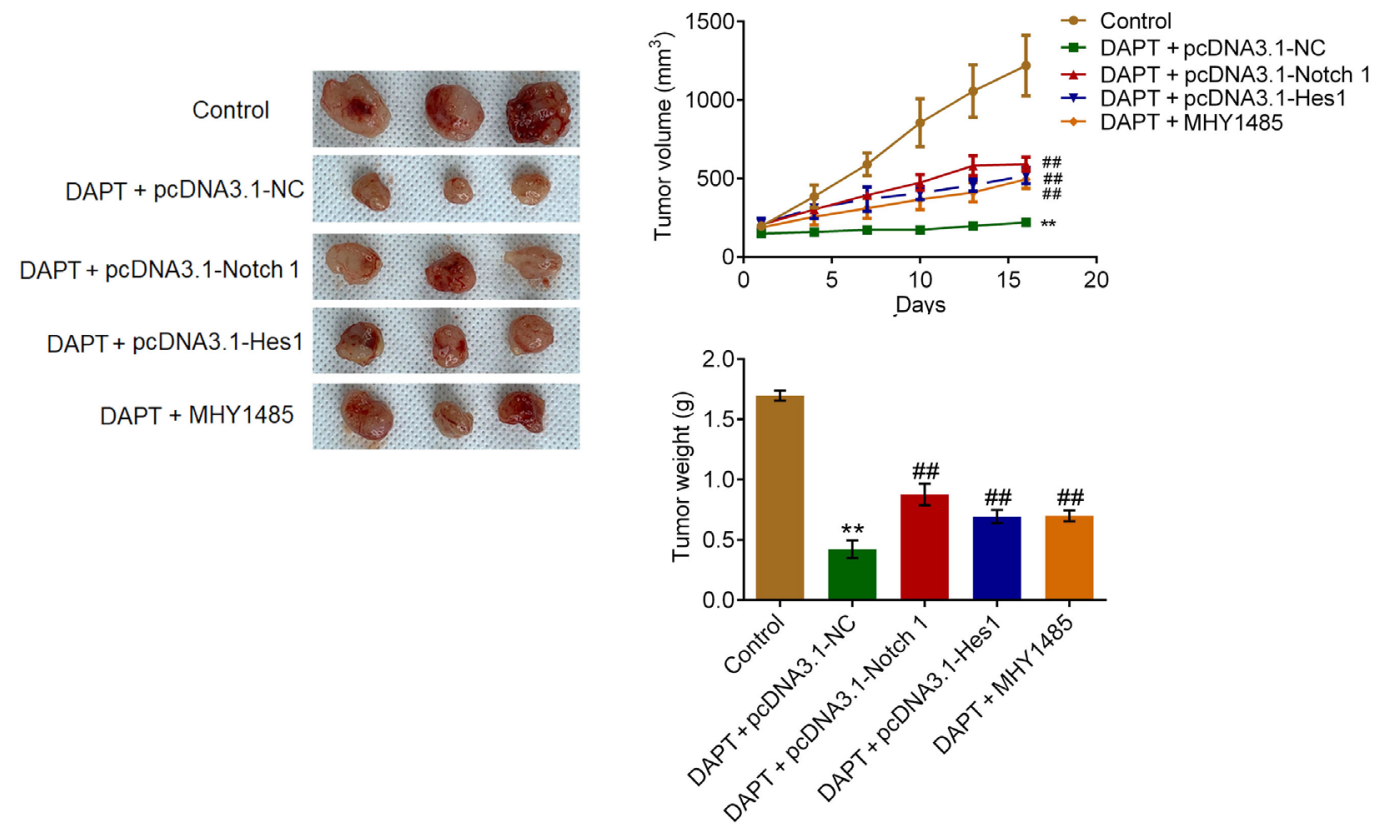

Figure $6 \mathrm{~N}$-(N-[3,5-Difluorophenacetyl]-1-alanyl)-S-phenylglycine t-butyl ester (DAPT) showed promising antitumor properties in a HepG2 xenograft model. Tumors were imaged, tumor volume was recorded every 3 days, and tumor weights were recorded after isolation. ${ }^{* *} \mathrm{P}<0.01$ vs. control, ${ }^{\# \#} \mathrm{P}<0.01$ vs. DAPT+pcDNA3.1- negative control (NC).

been reported to be involved in the regulation of cellular differentiation and tissue development, and the expression level of the Hes protein is regarded as an important marker for the activation of the Notch signaling pathway (23). Chen $e t$ al. recently reported that the activation of Notch1/ Hes1 signaling was involved in facilitating the progression of HCC (24). The Notch1 signaling pathway is also reported to be activated in HCC and contribute to the development and progression of HCC tumor formation (11). In the present study, we investigated the inhibitory effect of DAPT, a Notch1 inhibitor against HCC cell proliferation and migration. First, we confirmed that Notch1 was indeed highly expressed in both HCC tissues and cell lines. HepG2 cells were chosen as the experimental subject in the present study, as the expression level of Notch1 was found to be highest in the selected HCC cell lines. Subsequently, the in vitro antitumor effects of DAPT was evidenced by the decreased proliferation and migration ability, as well as the increased apoptotic rate. Further signaling investigations revealed that Notch1/Hes1 signaling was significantly blocked by DAPT, which was consistent with Zhang et al.'s finding (25). Promising antitumor properties were subsequently verified by the significantly decreased tumor volume in the DAPT-treated HepG2 xenograft animals.
To further confirm that the antitumor properties of DAPT were mediated by the Notch1/Hes 1 signaling pathway, we established the Notch1-overexpressed and Hes1overexpressed HepG2 cell lines. We found that the in vitro regulation of DAPT on the proliferation, migration, and apoptosis of HepG2 cells, and the in vivo tumor growth inhibitory effects, were reversed by the overexpression of Notch1 or Hes1. These data support the involvement of Notch1/Hes1 signaling in the antitumor properties of DAPT. In our future work, the impact of DAPT on more downstream molecules of Notch signaling will be investigated, such as eIF3a, SRY-related high-mobilitygroup-box protein-2 (SOX2), and Nrf2 to fully understand the antitumor mechanism of DAPT (26-28).

PTEN is an important tumor suppressor that has been reported to be downregulated in multiple types of malignant tumor cells (29). It has also been verified to have a low expression in HCC cells, and its degradation contributes to HCC progression (30). PTEN expression has been reported to be negatively regulated by Notch1/Hes1 signaling $(31,32)$. PTEN downregulation is regarded as an important inducer of the activation of the AKT/mTOR signaling pathway, which plays an important role in regulating the proliferation of multiple types of tumor cell lines $(33,34)$. 

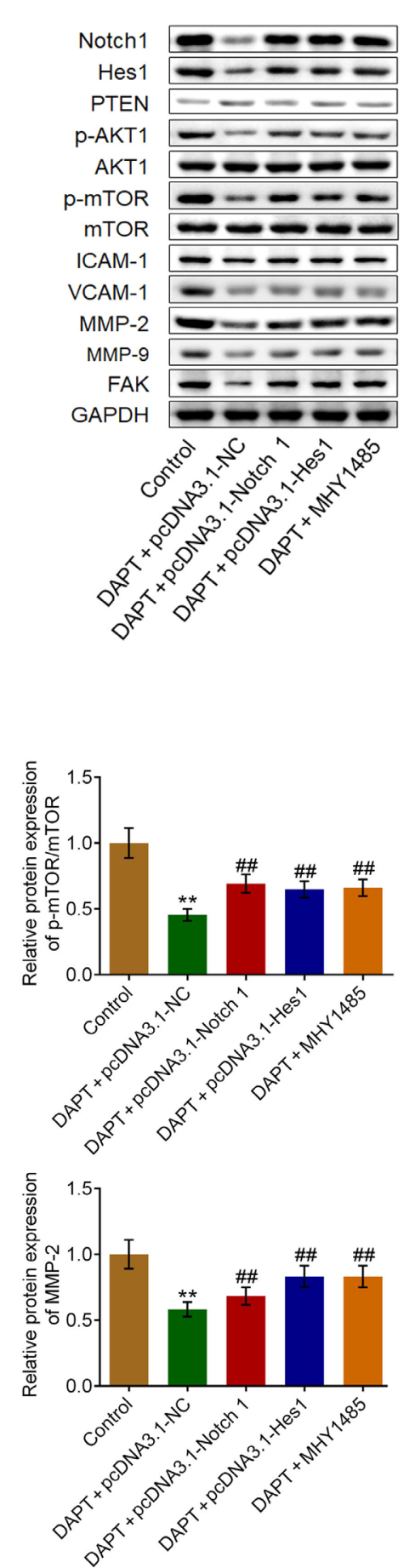
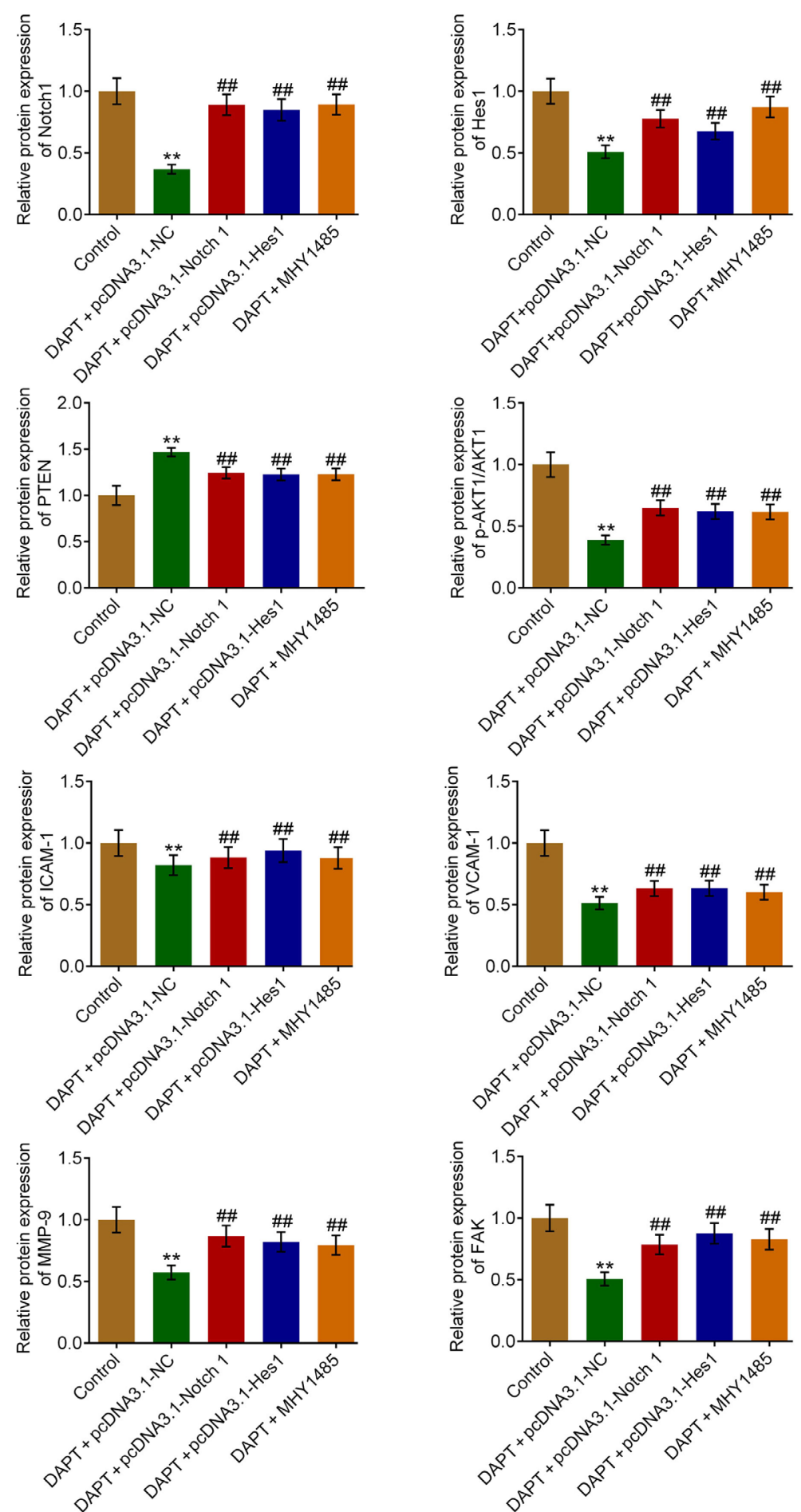

Figure 7 The effects of DAPT on Hes1/PTEN/AKT/mTOR signal pathway, ECM, and adhesion molecules in the xenograft tumor tissues were abolished by activating the Hes1/PTEN/AKT/mTOR signal pathway. The expression level of Notch1, Hes1, PTEN, AKT1, p-AKT1, mTOR, p-mTOR, ICAM-1, VCAM-1, MMP-2, MMP-9, and FAK in the tumor tissues was evaluated by Western blot assay. ** $<<0.01$ vs. control, ${ }^{\#} \mathrm{P}<0.01$ vs. DAPT+pcDNA3.1-negative control (NC). 
In the present study, we found that DAPT elevated the expression of PTEN by inhibiting Notch1/Hes1 signaling, further suppressing the AKT/mTOR signaling pathway. As a consequence, the proliferation and migration ability of HepG2 cells were inhibited, and the apoptotic rate of HepG2 cells increased. The effects of DAPT on Notch1/ Hes1/PTEN/AKT/mTOR signaling was further verified by introducing the activator of mTOR. In our future work, the PTEN knockdown of HepG2 cells will be introduced to further verify the signaling transduction route of DAPT.

The degradation of the ECM has been reported to be involved in the development and progression of tumor metastasis, which could be induced by the function of MMPs (35). In addition, the upregulation of adhesion molecules is also regarded as an important inducer for the migration of tumor cells (36). In the present study, we found that DAPT protected the ECM from degradation by downregulating MMP-2 and MMP-9, accompanied by the downregulation of adhesion molecules, which were all reversed by the activation of the Notch1/Hes1 signaling pathway or the activation of the AKT/mTOR signaling pathway. It is a novel finding that blocking Notch1/Hes1 signaling pathway suppressed the migration of tumor cells by preventing the ECM from degradation and downregulating adhesion molecules. Our future work will involve more detailed investigation into the regulatory effects of DAPT on the ECM and the expression of adhesion molecules to provide more support for the therapeutic application of DAPT in the treatment of HCC.

Taken together, our data indicated that DAPT could suppress the proliferation and migration of HCC by regulating the ECM and inhibiting the Hes1/PTEN/AKT/ mTOR signaling pathway.

\section{Acknowledgments}

Funding: This study was supported by Medicine and Health Science and Technology Plan Projects in Zhejiang Province (NO:2020RC108), Ningbo Natural Science Foundation (NO: 202003N4211) and Ningbo Clinical Medicine Research Center Project (grant NO: 2019A21003)

\section{Footnote}

Reporting Checklist: The authors have completed the ARRIVE reporting checklist. Available at http://dx.doi. org/10.21037/jgo-21-235
Data Sharing Statement: Available at http://dx.doi. org/10.21037/jgo-21-235

Conflicts of Interest: All authors have completed the ICMJE uniform disclosure form (available at http://dx.doi. org/10.21037/jgo-21-235). The authors have no conflicts of interest to declare.

Ethical Statement: The authors are accountable for all aspects of the work in ensuring that questions related to the accuracy or integrity of any part of the work are appropriately investigated and resolved. The study was conducted in accordance with the Declaration of Helsinki (as revised in 2013). The study was approved by Ethics Committee Board of Ningbo Medical Center Lihuili Hospital (NO: KY2020PJ190). Because of the retrospective nature of the study, the requirement for informed consent was waived. A protocol related to animal experiments was prepared before the study, which can be accessed by contacting the corresponding author. It was approved by the Animal Ethics committee of Ningbo University School of Medicine (NO: 2019-381) and guidelines for the care and use of animals were followed.

Open Access Statement: This is an Open Access article distributed in accordance with the Creative Commons Attribution-NonCommercial-NoDerivs 4.0 International License (CC BY-NC-ND 4.0), which permits the noncommercial replication and distribution of the article with the strict proviso that no changes or edits are made and the original work is properly cited (including links to both the formal publication through the relevant DOI and the license). See: https://creativecommons.org/licenses/by-nc-nd/4.0/.

\section{References}

1. Villanueva A. Hepatocellular Carcinoma. N Engl J Med 2019;380:1450-62.

2. Hu X, Yang X, He Q, et al. Glyoxalase 1 is up-regulated in hepatocellular carcinoma and is essential for HCC cell proliferation. Biotechnol Lett 2014;36:257-63.

3. Song P, Feng X, Zhang K, et al. Screening for and surveillance of high-risk patients with HBV-related chronic liver disease: promoting the early detection of hepatocellular carcinoma in China. Biosci Trends 2013;7:1-6.

4. Morimoto M, Numata K, Kondo M, et al. Radiofrequency 
ablation combined with transarterial chemoembolization for subcapsular hepatocellular carcinoma: a prospective cohort study. Eur J Radiol 2013;82:497-503.

5. Chung W, Kim M, de la Monte S, et al. Activation of signal transduction pathways during hepatic oncogenesis. Cancer Lett 2016;370:1-9.

6. Bray SJ. Notch signalling in context. Nat Rev Mol Cell Biol 2016;17:722-735.

7. Lee HJ, Kim MY, Park HS. Phosphorylation-dependent regulation of Notch1 signaling: the fulcrum of Notch1 signaling. BMB Rep 2015;48:431-7.

8. Hany H, Shalaby A, Al Kashef W, et al. Evaluation of the role of Notch 1 expression in hepatic carcinogenesis with clinico-pathological correlation. Pathology 2018;50:730-6.

9. Wang Z, Azmi AS, Ahmad A, et al. TW-37, a smallmolecule inhibitor of Bcl-2, inhibits cell growth and induces apoptosis in pancreatic cancer: involvement of Notch-1 signaling pathway. Cancer Res 2009;69:2757-65.

10. Zhang Y, Li D, Feng F, et al. Progressive and Prognosis Value of Notch Receptors and Ligands in Hepatocellular Carcinoma: A Systematic Review and Meta-analysis. Sci Rep 2017;7:14809.

11. Villanueva A, Alsinet C, Yanger K, et al. Notch signaling is activated in human hepatocellular carcinoma and induces tumor formation in mice. Gastroenterology 2012;143:1660-9.e7.

12. Fu H, Xing F, Lv Y, et al. ICBP90 mediates Notch signaling to facilitate human hepatocellular carcinoma growth. Tissue Cell 2018;54:65-71.

13. Zhang HF, Li W, Han YD. LINC00261 suppresses cell proliferation, invasion and Notch signaling pathway in hepatocellular carcinoma. Cancer Biomark 2018;21:575-82.

14. Lu L, Liu S, Dong Q, et al. Salidroside suppresses the metastasis of hepatocellular carcinoma cells by inhibiting the activation of the Notch1 signaling pathway. Mol Med Rep 2019;19:4964-72.

15. Fender AW, Nutter JM, Fitzgerald TL, et al. Notch-1 promotes stemness and epithelial to mesenchymal transition in colorectal cancer. J Cell Biochem 2015;116:2517-27.

16. Zhong JH, Zhou HJ, Tang T, et al. Activation of the Notch-1 signaling pathway may be involved in intracerebral hemorrhage-induced reactive astrogliosis in rats. J Neurosurg 2018;129:732-9.

17. Bui QT, Im JH, Jeong SB, et al. Essential role of Notch4/ STAT3 signaling in epithelial-mesenchymal transition of tamoxifen-resistant human breast cancer. Cancer Lett
2017;390:115-25.

18. Geng N, Jin Y, Li Y, et al. AKR1B10 Inhibitor Epalrestat Facilitates Sorafenib-Induced Apoptosis and Autophagy Via Targeting the mTOR Pathway in Hepatocellular Carcinoma. Int J Med Sci 2020;17:1246-56.

19. Li L, Tang P, Li S, et al. Notch signaling pathway networks in cancer metastasis: a new target for cancer therapy. Med Oncol 2017;34:180.

20. Artavanis-Tsakonas S, Rand MD, Lake RJ. Notch signaling: cell fate control and signal integration in development. Science 1999;284:770-6.

21. Iso T, Sartorelli V, Poizat C, et al. HERP, a novel heterodimer partner of $\mathrm{HES} / \mathrm{E}(\mathrm{spl})$ in Notch signaling. Mol Cell Biol 2001;21:6080-9.

22. Maier MM, Gessler M. Comparative analysis of the human and mouse Hey1 promoter: Hey genes are new Notch target genes. Biochem Biophys Res Commun 2000;275:652-60.

23. Nickoloff BJ, Osborne BA, Miele L. Notch signaling as a therapeutic target in cancer: a new approach to the development of cell fate modifying agents. Oncogene 2003;22:6598-608.

24. Chen Z, Zuo X, Pu L, et al. Hypomethylation-mediated activation of cancer/testis antigen KK-LC-1 facilitates hepatocellular carcinoma progression through activating the Notch1/Hes1 signalling. Cell Prolif 2019;52:e12581.

25. Zhang H, Jiang H, Chen L, et al. Inhibition of Notch1/ Hes1 signaling pathway improves radiosensitivity of colorectal cancer cells. Eur J Pharmacol 2018;818:364-70.

26. Gao YX, Jiang LL, Zhang Q, et al. Rutaecarpine protects against bleomycin-induced pulmonary fibrosis through inhibiting Notch1/eIF3a signaling pathway in rats. Zhongguo Zhong Yao Za Zhi 2018;43:3530-8.

27. Seo EJ, Kim DK, Jang IH, et al. Hypoxia-NOTCH1SOX2 signaling is important for maintaining cancer stem cells in ovarian cancer. Oncotarget 2016;7:55624-38.

28. Zhou XL, Wu X, Zhu RR, et al. Notch1-Nrf2 signaling crosstalk provides myocardial protection by reducing ROS formation. Biochem Cell Biol 2020;98:106-11.

29. Jiang Y, Liao HY, Yang QS, et al. Exploration of the Role of Tumor Suppressor Genes Foxo1 and PTEN in the Tumorigenesis of Mouse Natural Killer-Cell Lymphoma. Zhongguo Shi Yan Xue Ye Xue Za Zhi 2019;27:439-44.

30. Liu L, Long H, Wu Y, et al. HRD1-mediated PTEN degradation promotes cell proliferation and hepatocellular carcinoma progression. Cell Signal 2018;50:90-9.

31. Liu X, Zhang Y, Shi M, et al. Notch1 regulates PTEN expression to exacerbate renal tubulointerstitial fibrosis 
in diabetic nephropathy by inhibiting autophagy via interactions with Hes1. Biochem Biophys Res Commun 2018;497:1110-6.

32. Li X, Zou F, Lu Y, et al. Notch1 contributes to TNFalpha-induced proliferation and migration of airway smooth muscle cells through regulation of the Hes1/ PTEN axis. Int Immunopharmacol 2020;88:106911.

33. Qin J, Fu M, Wang J, et al. PTEN/AKT/mTOR signaling mediates anticancer effects of epigallocatechin3 gallate in ovarian cancer. Oncol Rep 2020;43:1885-96.

34. Liu S, Jia J, Zhou H, et al. PTEN modulates neurites

Cite this article as: Qiu K, Ma C, Lu L, Wang J, Chen B, Mao H, Wang Y, Wang H. DAPT suppresses proliferation and migration of hepatocellular carcinoma by regulating the extracellular matrix and inhibiting the Hes1/PTEN/AKT/ mTOR signaling pathway. J Gastrointest Oncol 2021;12(3):11011116. doi: 10.21037/jgo-21-235 outgrowth and neuron apoptosis involving the PI3K/Akt/mTOR signaling pathway. Mol Med Rep 2019;20:4059-66.

35. Walker C, Mojares E, Del Rio Hernandez A. Role of Extracellular Matrix in Development and Cancer Progression. Int J Mol Sci 2018;19:3028.

36. Moh MC, Shen $\mathrm{S}$. The roles of cell adhesion molecules in tumor suppression and cell migration: a new paradox. Cell Adh Migr 2009;3:334-6.

(English Language Editor: R. Scott) 